\title{
Evaluation of a Passive Air Sampler for Measuring Indoor Formaldehyde
}

\author{
Sun-Tae KIM, ${ }^{* \dagger}$ Bongbeen YIM,** and Jaeho JEONG* \\ *Department of Environmental Engineering, College of Engineering, Daejeon University, \\ 96-3, Yongun-dong, Dong-gu, Daejeon 300-716, Korea \\ **R \& D Center, Envors Co., Ltd., 151-13, Birae-dong, Daeduk-gu, Daejeon 306-809, Korea
}

\begin{abstract}
A passive air sampler, using 4-amino-3-hydrazino-5-mercapto-1,2,4-triazole, was evaluated for the determination of formaldehyde in indoor environments. Chromatography paper cleaned using a 3\% hydrogen peroxide solution was experimentally determined as being the optimum absorption filter for the collection of formaldehyde $\left(0.05 \mu \mathrm{g} \mathrm{cm}{ }^{-2}\right.$ formaldehyde). From a linear-regression analysis between the mass of formaldehyde time-collected on a passive air sampler and the formaldehyde concentration measured by an active sampler, the sampling rate of the passive air sampler was $1.52 \mathrm{~L} \mathrm{~h}^{-1}$. The sampling rate, determined for the passive air sampler in relation to the temperature $\left(19-28^{\circ} \mathrm{C}\right)$ and the relative humidity $(30-90 \%)$, were $1.56 \pm 0.04$ and $1.58 \pm 0.07 \mathrm{~L} \mathrm{~h}^{-1}$, respectively. The relationship between the sampling rate and the air velocity was a linear-regression within the observed range. In the case of exposed samplers, the stability of the collected formaldehyde decreased with increasing storage time (decrease of $c a$. 25\% after 22 days); but with the unexposed samplers the stability of the blank remained relatively unchanged for 7 days (decrease of $c a .37 \%$ after 22 days). The detection limits for the passive air sampler with an exposure time of 1 day and 7 days were 10.4 and $1.48 \mu \mathrm{g} \mathrm{m}^{-3}$, respectively.
\end{abstract}

(Received November 15, 2006; Accepted January 12, 2007; Published April 10, 2007)

Formaldehyde is present in houses and buildings as an indoorgenerated pollutant. The emissions of formaldehyde, from a variety of sources, such as building materials, furniture, fiberglass insulation and paper products, are expected to persist over comparatively long periods. ${ }^{1}$

Recently, an increase in volatile chemicals, including formaldehyde has led to unhealthy symptoms of people who spend much of their time in sick houses and buildings, and consequently suffering from various health problems. $^{2}$ The concentration of formaldehyde measured in workplaces ranged from 21.6 to 61.2 ppbv. $^{3}$ The average concentrations of formaldehyde in living rooms and bedrooms were 37 and $46 \mu \mathrm{g}$ $\mathrm{m}^{-3}$, respectively. ${ }^{4}$ The Korean indoor guideline for formaldehyde in various indoor environments is $120 \mu \mathrm{g} \mathrm{m}^{-3}$.

The sustained measurement of formaldehyde in indoor environments is of importance. In order to investigate the quality of indoor and ambient air, some methods for measuring formaldehyde have been developed using passive sampling techniques, employing the reaction of formaldehyde with 2,4dinitrophenylhydrazine (2,4-DNPH) reagent, 3-methyl-2benzothiazolinone hydrazone hydrochloride reagent, and chromotropic. ${ }^{3,5,6}$

Passive samplers have often been used to measure and evaluate the concentration levels of air pollutants because of their convenience in deployment and collection. Passive samplers require no power during sampling, and have no moving parts, such as pumps on flowmeters. The cost of passive samplers is likely to be low. They can be deployed almost anywhere to estimate the concentration levels of indoor air pollutants, although the real-time concentration data of pollutants can not be collected. The long-term effective concentrations obtained by passive sampling are also very

$\uparrow$ To whom correspondence should be addressed.

E-mail: envsys@dju.ac.kr useful in exposure assessment. ${ }^{7,8}$

In this study, we evaluated a passive air sampler using 4amino-3-hydrazino-5-mercapto-1,2,4-triazole (AHMT) reagent. We determined the effects of the sampling time, air velocity, air humidity, temperature, and storage period on the measurement of formaldehyde.

\section{Experimental}

\section{Chamber system and design of a passive air sampler}

A chamber system, with a badge-type passive air sampler, was prepared for the generation and measurement of formaldehyde. Passive air samplers were evaluated in a cylindrical exposure chamber $\left(0.02 \mathrm{~m}^{3}\right)$, which employed a mixing fan, and sensors (TSI, Model 8386, USA) for monitoring the temperature, humidity and air velocity. The concentration of formaldehyde, which was generated from formaldehyde solution (36.5-38\% in water, Sigma-Aldrich) by introduction with pure nitrogen gas (>99.99\%), was measured by employing an active sampling technique, using a portable sample pump with a flow rate of 0.5 $\mathrm{L} \mathrm{min}^{-1}$ and an absorption solution ( $0.5 \%$ boric acid solution).

Figure 1 shows a schematic diagram of the passive air sampler employed in this study, which consisted of a sampler end cap $(55 \times 55 \times 18 \mathrm{~mm})$, a sampler body (36-mm i.d.), a high-density polyethylene diffusion screen (33-mm i.d.), a semi-permeable membrane (Celgard-2500, micro porous polypropylene membrane; porosity, 55\%; pore size, $0.209 \times 0.054 \mu \mathrm{m}$; thickness, $25 \mu \mathrm{m}$, USA) and an absorption filter (pure cellulose chromatography paper; thickness, $0.17 \mathrm{~mm}, 26 \mathrm{~mm}$; Whatman, England). The diffusion length of the sampler was able to be changed from 4.5 to $22 \mathrm{~mm}$ by varying the location of the absorption filter within the sampler. The passive air sampler was easy to handle due to the light, safe components employed.

Coated absorption filters were individually prepared by dropping $0.1 \mathrm{~mL}$ of absorbing solution into the sampler body, with the 


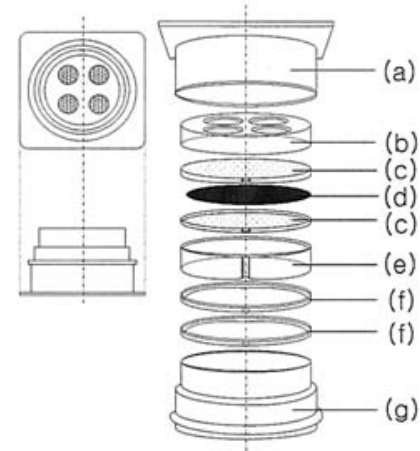

Fig. 1 Schematic diagram of the passive air sampler. (a) Upper cap, (b) diffusion barrier, (c) semi-permeable membrane, (d) absorption filter, (e) spacer, (f) support ring, (g) bottom cap.

components of passive air sampler then immediately assembled. Particular attention was given in the handling of samplers, because they can be easily contaminated. All passive air samplers were stored at $4^{\circ} \mathrm{C}$ in sealed bags in darkness until required.

\section{Analysis}

After sampling, the absorption filter of the passive air sampler was extracted with $2 \mathrm{~mL}$ of pure water in a glass vial. After extraction, $2 \mathrm{~mL}$ of $5 \mathrm{M}$ sodium hydroxide and an AHMT solution $(0.5 \mathrm{~g}$ in $100 \mathrm{~mL}$ of $0.1 \mathrm{M}$ hydrochloric acid) were added to the extraction solution, with a potassium periodate solution $(0.75 \mathrm{~g}$ in $100 \mathrm{~mL}$ of $0.2 \mathrm{M}$ sodium hydroxide), as the oxidizing reagent, added to the extraction solution after a further $20 \mathrm{~min}$. The colored solution had a maximum absorbance value of approximately $550 \mathrm{~nm}$. In the same manner, the absorption solution of the active sampling technique was analyzed spectrophotometrically using AHMT and an oxidizing reagent., 9,10

The electric current, measured by a colorimeter with a colored solution, was compared with the absorbance at $550 \mathrm{~nm}$ obtained using a spectrophotometer (U-1800, Hitachi). The linearity between the electric current and absorbance was good $\left(r^{2}=\right.$ 0.9996), and the linear response to the absorbance appeared to be satisfactorily within the range of the electric current $(\sim 50$ $\mu \mathrm{A})$ of the colorimeter. The electric current values of the sample were quantified from a linear-regression line fitted for standard solutions prepared from serial dilutions of a formalin solution. The concentrations of calibration standards ranged from 0 to $400 \mu \mathrm{g} \mathrm{L}^{-1}$. The regression equation was $Y$ (formaldehyde concentration, $\mu \mathrm{g} \mathrm{L}^{-1}$ ) $=3.2 X$ (electric current, $\mu \mathrm{A})+7.3$ with $n=12$ and $r^{2}=0.987$.

\section{Evaluation of passive air samplers}

The evaluation of the blank values for the formaldehyde passive air samplers was investigated employing a variety of absorption and different cleaning methods. All filters were cut into identical $2.4 \mathrm{~cm}$ diameter circles, extracted and then analyzed as described above. In addition, the procedures used for cleaning the absorption filters were investigated with pure water and oxidizing agents, like hydrogen peroxide. The absorption filters were cleaned by washing for $1 \mathrm{~h}$ in an ultrasonic cleaner $(40 \mathrm{kHz}, 1500 \mathrm{~W})$, and then completely dried in a drying oven $\left(60^{\circ} \mathrm{C}\right)$.

A comparison of the absorption efficiencies of two absorbent reagents was carried out employing two viscous liquids, namely triethanolamine $\left(\text { viscosity }=590.5 \mathrm{mPa} \mathrm{s}^{-1} \text { at } 25^{\circ} \mathrm{C}\right)^{11}$ and phosphoric acid (viscosity $=3.86 \mathrm{mPa} \mathrm{s}^{-1}$ at $20^{\circ} \mathrm{C}, 40 \%$ solution). ${ }^{12}$ The absorption filters, which were coated by 100 $\mu \mathrm{L}$ of triethanolamine $(10,20$, and $40 \%)$ and phosphoric acid $(5,10$, and $20 \%)$, were simultaneously exposed to formaldehyde gas $\left(c a .400 \mu \mathrm{g} \mathrm{m}^{-3}\right)$ in the chamber.

\section{Determination of passive sampling rate}

Passive and active sampling methods were carried out simultaneously in the exposure chamber to estimate the sampling rate for the determination of the formaldehyde concentration. The exposure experiment using the passive air sampler in the chamber was performed with concentrations ranging from 15 to $480 \mu \mathrm{g} \mathrm{m}^{-3}$. The temperature and relative humidity in the chamber throughout the exposure period were held constant at $25 \pm 3^{\circ} \mathrm{C}$ and $40 \pm 10 \%$, respectively. Four samplers were installed in the chamber, and exposed for periods of between 3 and $24 \mathrm{~h}$.

The mass of formaldehyde collected on the exposed passive air sampler was corrected by subtracting the average mass measured for the blanks. The reference formaldehyde concentration was determined from the mass of formaldehyde collected by the active sampler in relation to the volume of air drawn by the pump.

\section{Passive air sampler tests}

The temperature was controlled by a heating coil equipped in the chamber and a heating tape installed on a stainless-steel gas tube. The humidity was regulated by passing a portion of the pure nitrogen gas through pure water in several bubblers. The air velocity in the chamber was controlled by rotating the mixing fan, and was measured in the neighborhood of the inlet of the passive air sampler.

The experimental conditions were constantly monitored using temperature, relative humidity and air velocity probe-type multifunction sensors, which were selected so as to cover the temperature, relative humidity and air velocity ranges of 18 to $30^{\circ} \mathrm{C}, 30$ to $90 \%$ and 0 to $0.3 \mathrm{~m} \mathrm{~s}^{-1}$, respectively. To test the effect of one factor, other factors were fixed (temperature, $25 \pm$ $3^{\circ} \mathrm{C}$; relative humidity, $40 \pm 10 \%$; air velocity, $0.02 \mathrm{~m} \mathrm{~s}^{-1}$ ).

The stability of the exposed and unexposed passive air samplers with respect to the storage period in sealed bags with an intense opaque black color at room temperature $\left(25 \pm 5^{\circ} \mathrm{C}\right)$ was investigated. The precision of the measurement method using passive air sampler offers valuable information, and can best be obtained from multiple co-located measurements from samplers exposed to the same experimental conditions. Four replicate passive air samplers were investigated with the formaldehyde concentration ranging from $25-300 \mu \mathrm{g} \mathrm{m}^{-3}$ over a $24-\mathrm{h}$ period. All experiments were carried out at least in duplicate.

\section{Results and Discussion}

Evaluation of the collection filter and absorptions solution

The formaldehyde levels of the glass fiber and chromatography filter papers were $0.10 \pm 0.01$ and $0.17 \pm 0.01$ $\mu \mathrm{g} \mathrm{cm}^{-2}$ (mean \pm standard deviation), respectively. However, those of the qualitative filter papers (5A and 5C) were $2.41 \pm$ 0.11 and $2.26 \pm 0.15 \mu \mathrm{g} \mathrm{cm}^{-2}$, respectively. The quantity of formaldehyde contained in the glass fiber and chromatography filter papers were lower than those in the qualitative filter papers. Since glass fiber filter paper easily undergoes degradation during the extraction process, and due to the oxidizing reagent solutions, with a resultant increase in the turbidity of the analytical solution, chromatography filter papers were chosen and adopted throughout the course of this study owing to their reasonable cost.

After cleaning with $3 \%$ hydrogen peroxide, the quantity of formaldehyde obtained from chromatography filter paper was 


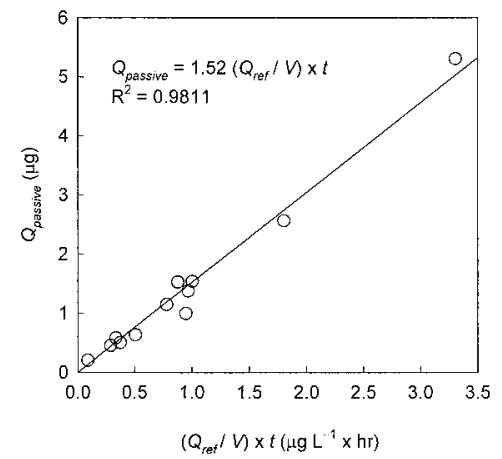

Fig. 2 Correlation between the time-corrected formaldehyde mass collected by the passive air sampler and formaldehyde concentration measured by the active sampler.

$0.05 \pm 0.01 \mu \mathrm{g} \mathrm{cm}^{-2}$, with a formaldehyde elimination of about $70.6 \%$ compared with no cleaning $\left(0.17 \pm 0.03 \mu \mathrm{g} \mathrm{cm}^{-2}\right)$. In the case of pure water, the quantity of obtained formaldehyde was $0.09 \pm 0.01 \mu \mathrm{g} \mathrm{cm}^{-2}$, with a formaldehyde removal of about $47 \%$; $3 \%$ hydrogen peroxide was found to be an effective cleaning agent for reducing the blank values obtained from the absorption filters used in the passive air samplers. However, the possibility of oxidation due to the hydrogen peroxide remaining in the absorption filters might be capable of affecting the color developed in solution. In this study, after cleaning the absorbance filters with $3 \%$ hydrogen peroxide, repetitive ultrasonic cleaning with pure water was additionally performed to minimize the effect of hydrogen peroxide, thus reducing this possible source of contamination.

The absorption efficiency of $40 \%$ triethanolamine solution on the collection of formaldehyde was highest $(6.6 \pm 1.23 \mu \mathrm{g}$ $\left.\mathrm{cm}^{-2}\right)$, followed by $20 \%$ triethanolamine $\left(6.4 \pm 0.73 \mu \mathrm{g} \mathrm{cm}^{-2}\right)$, $10 \%$ triethanolamine $\left(4.5 \pm 0.29 \mu \mathrm{g} \mathrm{cm}^{-2}\right)$, phosphoric acid $(\sim 1$ $\left.\mu \mathrm{g} \mathrm{cm}^{-2}\right)$ and finally pure water $\left(0.56 \pm 0.07 \mu \mathrm{g} \mathrm{cm}^{-2}\right)$. An increasing of the absorption efficiency at concentrations over $40 \%$ triethanolamine was little observed. The absorption efficiencies with triethanolamine solutions were found to be over four-times greater than those of the phosphoric acid solutions, and those of phosphoric acid solutions were independent of the concentration. The use of a viscous reagent as an absorption solution would seem to be most applicable for measuring formaldehyde in the atmosphere.

\section{Determination of the passive sampling rate}

The sampling rate of a passive air sampler can be experimentally determined by a direct comparison to the active sampling technique mentioned aboved. The sampling rate of the diffusive air sampler can be practically calculated using following equations: ${ }^{13}$

$$
\begin{aligned}
& \mathrm{SR}=\frac{Q_{\text {passive }} / t}{Q_{\text {ref }} / V}, \\
& Q_{\text {passive }}=\mathrm{SR} \cdot \frac{Q_{\text {ref }}}{V \cdot t},
\end{aligned}
$$

where SR is the sampling rate $\left(\mathrm{L} \mathrm{h}^{-1}\right), Q_{\text {passive }}$ the mass of the formaldehyde transported into passive air sampler by diffusion $(\mu \mathrm{g}), t$ the exposure time (h), $Q_{\text {ref }}$ the mass of the formaldehyde collected in the active sampler and $V$ the volume of air introduced by the pump to the active sampler $(\mathrm{L})$.

Figure 2 shows the relationship between the mass of

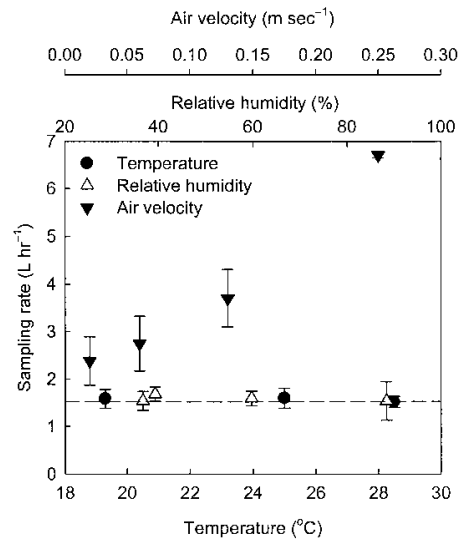

Fig. 3 Influence of the temperature, relative humidity and air velocity on the formaldehyde sampling rate. The sampling rate at 22 $\pm 3^{\circ} \mathrm{C}$ and $50 \pm 10 \%$ humidity was $1.52 \mathrm{~L} \mathrm{~min}^{-1}$ (dash line).

formaldehyde collected on the passive air sampler over time and the formaldehyde concentration measured by the active sampler, using a flow rate of $0.3 \mathrm{~L} \mathrm{~min}^{-1}$, in the chamber experiment. A linear-regression analysis between the two sampling methods gave a good correlation. The regression equation was $Q_{\text {passive }}=$ $1.52\left(Q_{\text {ref }} / V\right) \times t$ with a determination coefficient $\left(r^{2}\right)$ of 0.9811 . The sampling rate of the passive air sampler was $1.52 \mathrm{~L} \mathrm{~h}^{-1}$, as obtained from the slope of the linear correlation shown in Fig. 2. The mass of formaldehyde collected per hour on the passive air sampler was divided by the sampling rate to determine the formaldehyde concentrations measured by all of the other passive air samplers in the atmosphere.

\section{Effects of the temperature, relative humidity and wind speed}

Figure 3 shows the effect of changes in the sampling rates on the performance of the passive air sampler under certain experimental conditions. The sampling rates determined for the passive air sampler within adopted temperature and relative humidity ranges were $1.56 \pm 0.04$ and $1.58 \pm 0.07 \mathrm{~L} \mathrm{~h}^{-1}$, respectively. A decrease in the sampling rate of $0.4 \%{ }^{\circ} \mathrm{C}^{-1}$ was observed; the change of the sampling rate by relative humidity was less than $0.1 \%$. This would be due to partial drying of the absorption solution on the surface of the collection filter. A decrease in the sampling rate due to the effect of temperature has previously been observed on the performance of the passive air sampler for benzene. ${ }^{14}$ The determination coefficients found from a regression line between the sampling rate and temperature or the relative humidity were 0.472 and 0.216 , with slopes of only -0.006 and 0.001 , respectively. The slopes of the regression lines between the two variances were close to zero, indicating that the temperature and relative humidity had no significant effects on the sampling rates of the passive air sampler. The performance of the passive air sampler would be independent of the temperature and the relative humidity under above-mentioned experimental conditions.

The influence of the air velocity on the performance of the passive air sampler was also investigated, although dramatic variations in the air velocity in an indoor environment would not be often observed. Figure 3 also shows the effect of the air velocity on the sampling rate of the passive air sampler. A variation in the air velocity may cause a change in the diffusion path length at the front of the passive air sampler, because a static layer will be formed with a low air velocity in the vicinity of the inlet of the sampler, along with a reduction in the effective molecular diffusion length caused by a high air 


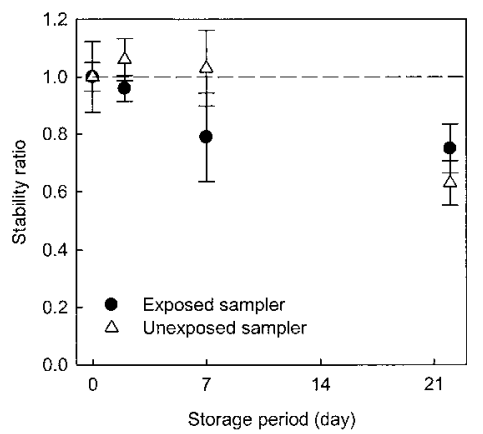

Fig. 4 Stability of exposed and unexposed passive air samplers as a function of the storage period. With a stability ratio of 1.0 , the quantities of formaldehyde collected in the exposed and unexposed passive air samplers were 2.5 and $0.5 \mathrm{~g}$, respectively.

velocity. ${ }^{15,16}$ The relationship between the sampling rate and the air velocity was a linear-regression within the observed range. A linear-regression analysis was carried out on our data, with the line of fit shown in Fig. 3, for sampling rate of $19.2 \times$ (air velocity) +1.6 . The determination coefficient for the regression analysis was 0.967 . The passive air sampler adopted in this study had a short diffusion length from the inlet of the sampler to the collection filter. The sampling rate was influenced by weak air velocities below $0.3 \mathrm{~m} \mathrm{~s}^{-1}$, even when a semipermeable membrane was installed on the collection filter to inhibit turbulence due to wind, and stabilize the molecular diffusion into the passive air sampler. If air turbulence occurs in the vicinity of the sampler inlet, the sampling rate could be increased; in addition, the concentration of formaldehyde in the atmosphere would be overestimated. However, the passive air sampler for measuring formaldehyde in this study was developed and evaluated for indoor environmental measurements, where the effects of air velocity and turbulence existed only slightly.

\section{Evaluation of the storage stability, precision and detection limits}

The formaldehyde concentrations of exposed and unexposed passive air samplers were measured immediately after assembly, giving value of 2.5 and $0.3 \mu \mathrm{g}$, respectively. As shown in Fig. 4, in the case of the exposed samplers, the stability of the formaldehyde collected was relatively decreased with increasing storage time: an approximate $25 \%$ decrease after 22 days (ratio $=0.75$ ). In the case of unexposed samplers, under the same storage conditions the stability of the blank remained reasonably unchanged for 7 days, but decreased to $37 \%$ after 22 days (ratio $=0.63$ ).

Table 1 gives the precisions of the results obtained for multiple co-located passive air samplers for several formaldehyde concentrations. The relative standard deviation derived from the measurements was found to be less than $10 \%$ (mean, 5.8\%; standard deviation, 2.4\%; range, $2.3-8.8 \%$ ). The precision of the passive air sampler used in this appeared to be independent of the formaldehyde concentration. Highly reproducible results were obtained, suggesting that the precision of the passive air samplers would be acceptable for measuring formaldehyde levels of indoor environments.

For exposure times of 1 day and 7 days, the mean blank concentrations calculated from the mass of formaldehyde on the unexposed passive air samplers $(n=7)$ were 8.7 and $1.3 \mu \mathrm{g} \mathrm{m}^{-3}$. The DL (QL) values of the passive air sampler were 10.4 (13.9) and 1.48 (1.99) $\mu \mathrm{g} \mathrm{m}^{-3}$, respectively. For measuring formaldehyde at lower concentrations, the exposure time should be increased.
Table 1 Precision of passive air samplers for measuring indoor formaldehyde levels

\begin{tabular}{rrrrrrrr}
\hline No. & \multicolumn{1}{c}{ Concentration/ $\mu \mathrm{g} \mathrm{m}^{-3}$} & $\begin{array}{r}\mathrm{Mean} / \\
\mu \mathrm{g} \mathrm{m}^{-3}\end{array}$ & $\begin{array}{c}\mathrm{SD}^{\mathrm{a} /} \\
\mu \mathrm{g} \mathrm{m}^{-3}\end{array}$ & $\begin{array}{c}\mathrm{RSD}^{\mathrm{b}} \\
\%\end{array}$ \\
\hline 1 & 27.5 & 27.5 & 26.6 & 26.3 & 27.0 & 0.6 & 2.3 \\
2 & 46.4 & 44.7 & 43.4 & 49.8 & 46.1 & 2.8 & 6.0 \\
3 & 93.3 & 103.6 & 105.6 & 99.6 & 100.5 & 5.4 & 5.4 \\
4 & 182.9 & 168.3 & 200.6 & 204.4 & 189.1 & 16.7 & 8.8 \\
5 & 276.6 & 260.2 & 291.2 & 303.2 & 282.8 & 18.6 & 6.6 \\
\hline
\end{tabular}

a. SD: standard deviation.

b. RSD: relative standard deviation.

Passive air samplers using AHMT reagent are well suited to indoor and personal monitoring, because they have the advantage of adequate precision, sample stability and ease of operation. Passive air samplers would also be suitable for both long-term measurements in ambient air with low pollutant concentrations, and for short-term measurements involving personal monitoring in workplaces with high pollutant concentrations.

\section{Acknowledgements}

This study was supported by the Ministry of Environment as "The Eco-technopia 21 Project".

\section{References}

1. D. R. Crump and D. Gardiner, Environ. Int., 1989, 15, 455.

2. T. Godish, "In Indoor Air Quality Handbook: Aldehydes", ed. J. D. Spengler, J. M. Samet, and J. F. McCarthy, 2001, McGraw-Hill, NY, 32.1 - 32.19.

3. R. W. Gillett, H. Kreibich, and G. P. Ayers, Environ. Sci. Technol., 2000, 34, 2051.

4. C. Marchand, B. Bulliot, S. Le Calve, and $\mathrm{Ph}$ Mirabel, Atmos. Environ., 2006, 40, 1336.

5. J. O. Levin, K. Andersson, R. Lindahl, and C. A. Nilsson, Anal. Chem., 1985, 57, 1032.

6. J. P. Lodge, "Methods of Air Sampling and Analysis", 3rd ed., 1988, Lewis Publishers, Chelsea, MI, 274 - 284.

7. N. L. Bernard, M. J. Gerber, C. M. Astre, and M. J. Saintot, Environ. Sci. Technol., 1999, 33, 217.

8. T. Gorecki and J. Namiesnik, Trends Anal. Chem., 2002, $21,276$.

9. R. G. Dickinson and N. W. Jacobsen, J. Chem. Soc. D: Chem. Commun., 1970, 24, 1719.

10. The Pharmaceutical Society of Japan, "Methods of Analytical in Health Science", 2000, Kanehara, Tokyo, 1451 - 1452.

11. M. J. O'Neil, "The Merck Index-An Encyclopedia of Chemicals, Drugs, and Biologicals", 13th ed., 2001, Merck and Co., Inc., Whitehouse Station, NJ, 1723.

12. Environment Canada, "Tech. Info. for Problem Spill: Phosphoric Acid", 1982, Environment Canada, Ottawa, 4.

13. N. E. Rabaud, T. A. James, L. L. Ashbaugh, and R. G. Flocchini, Environ. Sci. Technol., 2001, 35, 1190.

14. A. Pennequin-Cardinal, H. Plaisance, N. Locoge, O. Ramalho, S. Kirchner, and J.-C. Galloo, Atmos. Environ., 2005, 39, 2535.

15. A. J. Gair and S. A. Penkett, Atmos. Environ., 1995, 29, 2529.

16. J. Ballach, B. Greuter, E. Schultz, and W. Jaeschke, Sci. Total Environ., 1999, 243/244, 203. 